\title{
Synthesis of Polymer Sensor for Detection of Phosphate in Water
}

\author{
Worawit Wongniramaikul and Aree Choodum
}

\begin{abstract}
A colorimetric polymer sensor was successfully developed for the detection of phosphate in water. Potassium antimonyl tartrate, ammonium molybdate, ascorbic acid and sulfuric acid were entrapped within the polyvinyl alcohol (PVA) hydrogel matrix and acted as the colorimetric reagent. The PVA sensor was fabricated within a micro-PCR tube to which the sample solution could be directly added for in-tube detection. This made the developed sensor small and easy to carry on field. The optimum condition for sensor synthesis was determined in this research by using the digital image analysis. They are found at $0.6 \mathrm{~g} / \mathrm{L}$ potassium antimonyl tartrate, 14.4 $\mathrm{g} / \mathrm{L}$ ammonium molybdate, $5.28 \mathrm{~g} / \mathrm{L}$ ascorbic acid, $0.05 \mathrm{mg} / \mathrm{L}$ sulfuric acid and $15 \mathrm{~min}$ reaction time.
\end{abstract}

Index Terms-Hydrogel sensor, phosphate detection, polymer sensor.

\section{INTRODUCTION}

Classified as phosphorus compound, phosphate naturally appears in several forms, e.g. organic phosphate and polyphosphate. Nevertheless, the most bioavailable species is orthophosphate, $\mathrm{PO}_{4}{ }^{3-}, \mathrm{HPO}_{4}{ }^{2-}$ and $\mathrm{H}_{2} \mathrm{PO}_{4}{ }_{4}^{-}$, etc. [1]. It is also most abundant one, since it can be stabilized at neutral $\mathrm{pH}$ [2] The key point source for phosphate are the municipal wastewater [3] and the application as corrosion inhibitor in the wastewater treatment system [4], while the gigantic amount of phosphate released to the environment comes from the non-point source, such as agricultural fertilizer and livestock excretion [5], [6]. High concentration of phosphate in water body, especially a closed or semi-closed to drainage, causes the eutrophication [7]-[10]. This phenomenon is responsible for the sensational bloom of algae leading to the considerable drop of dissolved oxygen in a natural water resource. In addition to the environmental problem, the excess phosphate takes account to the progressive fouling of pipeline in the industrial water system [11].

To reduce these problems, the enough qualitative and quantitative information of the contaminated phosphate is required through the high efficient analytical method. Nowadays, various contemporary techniques are taken account to measure the phosphate in the collected sample, such as the high performance liquid chromatography [12] and ion chromatography [13]. These instrumental methods possess the advantage of good system performance,

Manuscript received January 15, 2017; revised April 15, 2017. This work was supported by the Faculty of Technology and Environment, Prince of Songkla University, Phuket Campus and Center of Excellence on Hazardous Substance Management (HSM), Chulalongkorn University.

Worawit Wongniramaikul and Aree Choodum are with the Faculty of Technology and Environment, Prince of Songkla University, Phuket Campus, Phuket 83120 Thailand (e-mail: worawit.won@phuket.psu.ac.th, aree.c@phuket.psu.ac.th). including high precision and low detection limit. However, the use of these techniques is prohibited by an investment cost of analytical instrument. The cheaper technique, spectrophotometry become more popular [5]. Other attractive reasons are simple-to-use and small space required. Even though, the tedious works of sampling and transferring the sample from a site to laboratory for analysis are inevitable by the technique. These also cause sample losses and contamination.

A colorimetric sensor would be an advantageous choice for rapid cost-effective on-site determinations of formaldehyde. This approach would provide a number of advantages such as field portability, visual qualitative feedback, rapid detection, and ease of handling [14], [15]. It is typically based on entrapment or impregnation of a colorimetric reagent within various materials, such as a sol-gel matrix [16], porous glass [14], or molecularly imprinted polymer [17].

PVA hydrogel is a macromolecule three-dimensional crosslinked PVA, which is capable of absorbing and retaining large amount of water and biological fluid [18]. It could be formed a stimulus-sensitive material by incorporating necessary functional groups. It has been used for various applications such as tissue engineering, drug delivery system, and biomedical engineering.

The PVA hydrogel polymer can be synthesized by either a chemical or physical cross-linking process [19]. Chemical cross-linking can be conducted by irradiation or the use of bifunctional cross-linkers, while physical cross-linking can be achieved by the use of freezing/thawing cycles to form crystals. The combination of the two methods of synthesis produces an elastic and sponge-like cryogel with good mechanical stability and high flow-through pores [20], [21].

In this article, a low cost colorimetric sensor was developed based on the synthesis of PVA hydrogel polymer. It was used incorporately with the digital image colorimetry by webcam for semi-quantitative analysis to deliver reliable results removing the necessity for spectrophotometric detection.

\section{MATERIALS AND METHODS}

\section{A. Materials}

All reagents were of analytical grade and the freshly de-ionized of $18 \mathrm{M} \Omega / \mathrm{cm}$ (Millipore, Bedford, MA, USA) was handled for preparing all solutions at desired concentration. A component for polymer synthesis, which were poly vinyl alcohol (Mw 85000-124000 g/mol, >99\% hydrolyzed) and poly ethylene glycol diglycidyl ether (EGDE, 98\%) were obtained from Sigma-Aldrich Ltd. (USA). A chromogenic species, which were ammonium 
molybdate tetrahydrate $\left(\left(\mathrm{NH}_{4}\right)_{6} \mathrm{Mo}_{7} \mathrm{O}_{24} \cdot 4 \mathrm{H}_{2} \mathrm{O}\right)$, potassium antimonyl tartrate hemihydrates $\left(\mathrm{K}(\mathrm{SbO}) \mathrm{C}_{4} \mathrm{H}_{4} \mathrm{O}_{6} \cdot 0.5 \mathrm{H}_{2} \mathrm{O}\right)$ and L-ascorbic acid $\left(\mathrm{C}_{6} \mathrm{H}_{8} \mathrm{O}_{6}\right)$, were purchased from Fisher Scientific (Leicestershire, UK), while the targeted analyte, potassium dihydrogen phosphate $\left(\mathrm{KH}_{2} \mathrm{PO}_{4}\right)$ was obtained from Carlo Erba (Val de Reuil , France). Other chemicals used in the experiment were concentrated sulfuric acid $\left(\mathrm{H}_{2} \mathrm{SO}_{4}\right)$ (J.T. Baker, New Jersey, USA).

\section{B. Preparation of the PVA Hydrogel Phosphate Sensor}

The PVA cryogel phophate sensor was prepared following the procedure reported by previous research [20]-[22] with some modification. In brief, PVA granule was dissolved in ultrapure water $(5 \% \mathrm{w} / \mathrm{v})$ at $120^{\circ} \mathrm{C}$ and stirred for $2 \mathrm{hr}$ to obtain a clear viscous solution. This solution was cooled at room temperature before further use. The colorimetric reagents, ammonium molybdate tetrahydrate, potassium antimonyl tartrate, ascorbic acid, and sulfuric acid under the optimized concentration were added to the PVA solution and stirred for $5 \mathrm{~min}$. Finally, $100 \mu \mathrm{L}$ EGDE was added as a cross-linker and the solution was stirred for a while. The polymer mixture of $130 \mu \mathrm{L}$ was then transferred into micro-PCR tubes and kept in a freezer overnight prior to further use.

For optimization of the sensor production, various concentration of necessary ingredients, including potassium antimonyl tartrate $(0.2-4.0 \mathrm{~g} / \mathrm{L})$, ammonium molybdate $(3.6$ - $57.6 \mathrm{~g} / \mathrm{L})$, ascorbic acid (0.88 - $10.56 \mathrm{~g} / \mathrm{L})$ and sulfuric acid $(0.02-0.11 \mathrm{mg} / \mathrm{L})$, was added into $5 \mathrm{~mL}$ PVA solution The optimum hydrogel recipe was chosen as that which produced the darkest color product of phosphate using the least amount of chemicals. The effect of reaction time was investigated on this stage of work as well.

\section{RGB Capturing System}

Since the principle of digital image analysis was based on the measurement of light reflection from the reaction product, the 3 " $\times 3.5 " \times 3.5$ " laboratory-built protective box was set up to limit the detection interference from the environmental light, especially sunlight. The box was made from an opaque black corrugated plastic board and interior coated with white background. The PCR micro-tube was placed on the top of protective box. Three light emitting-diodes (LEDs) were employed as the light source and the electrical power was supplied by the laptop computer with a USB cable connection. Front side of the box was perforated for a webcam installation. The digital photograph was obtained by Compaq Presario CQ40, WXGA $(1280 \times 800)$ High-definition Bright View LCD panel (16:10 Wide Aspect Ratio) equipped with webcam $640 \times 480$ pixels. The analysis of RGB intensity was performed by our colleague-built program based on MATLAB.

\section{RESULTS AND DISCUSSION}

This work reports the successful synthesis of a PVA hydrogel polymer as a low cost colorimetric sensor for the simple and rapid phosphate detection. The PVA sensor was formed using a combination of a chemical cross-linking process and cryogenic treatment. Ethylene glycol diglycidyl ether (EGDE) was used as the chemical cross-linker, while the colorimetric reagent was employed following the standard method [23] for phosphate detection. Entrapped colorimetric species was released from the hydrogel matrix to react with phosphate in the sample producing a specific color product. The optimum condition of sensor synthesis was determined through digital image colorimetry using a webcam.

\section{A. Effect of Colorimetric Reagent and Acidified Reagent}

The major problem of many field analytical techniques was an application of expensive chemicals. Thus, the portable sensor would be more attractive for the user, if a portion of all chemicals in those sensors were minimized. Furthermore, it could be answering the question of a green chemistry as well. On this ground, the concentration of colorimetric reagents for the sensor synthesis, including their effect on the color intensity, were investigated in this work. Experimental results were presented in Fig. 1-Fig. 4.

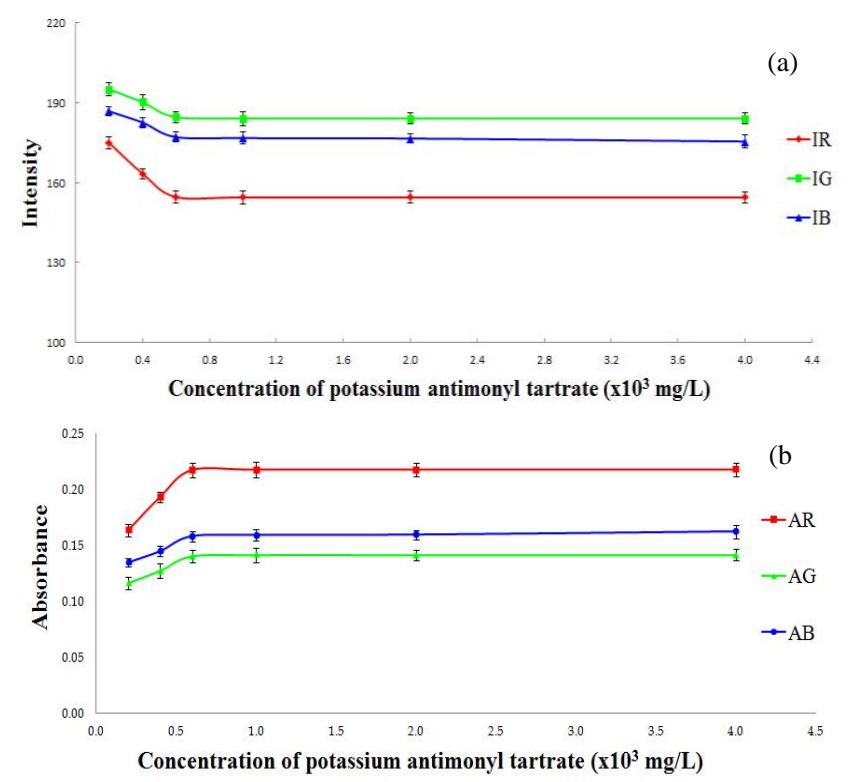

Fig. 1. The effect of potassium antimonyl tartrate concentration in sensor on (a) the RGB intensity and (b) absorbance for $1 \mathrm{mg} / \mathrm{L}$ phosphate detection.

One of colorimetric reagent, potassium antimonyl tartrate was added into the PVA sensor in the form of mixed reagent. The relationship between its concentration and the color intensity was shown in Fig. 1.

According to Fig. 1(a), it was clear that the intensity of Red $\left(\mathrm{I}_{\mathrm{R}}\right)$, Green $\left(\mathrm{I}_{\mathrm{G}}\right)$ and Blue $\left(\mathrm{I}_{\mathrm{B}}\right)$ were inversely proportional to the concentration on of potassium antimonyl tartrate in the range $0.2-0.6 \mathrm{~g} / \mathrm{L}$. This was due to more reaction product formation with increasing reactant, the color intensity was decreased. However, the intensity became constant after 0.6 $\mathrm{g} / \mathrm{L}$ pointing out the stabilized product. Consequently, it could be stated that the appropriate potassium antimonyl tartrate concentration for sensor synthesis should be $0.6 \mathrm{~g} / \mathrm{L}$ for $1 \mathrm{mg} / \mathrm{L}$ phosphate detection.

Because the eye sees the converse of the color component that was primarily absorbed. The molecular absorption of color products was also investigated by calculating the absorbance at each concentration using (1):

$$
A_{X}=-\log \frac{\left(I_{X}-I_{X, b}\right)}{\left(I_{x, w}-I_{x, b}\right)}=-\log \frac{\left(I_{x}\right)_{c}}{\left(I_{x, w}\right)_{c}}=-\log R_{x}
$$

where for each colour (R, B, G), $A_{X}$ was the absorbance of $X, I_{X}$ was the intensity of $X, \mathrm{I}_{X, b}$ was the intensity of black 
$\left(I_{X, b}=0\right), I_{X, w}$ was the intensity of white $\left(I_{X, w}=255\right)$, and $R_{X}$ was the reflectance of light $X$ and $C$ was the concentration of $X$ [24].

The logarithmic relationship for each color was presented in Figure 1(b). The obtained profiles were similar with those commonly reported using spectrophotometric methods.

In contrast with the intensity, the RGB absorbance was increased with the increasing potassium antimonyl tartrate concentration (Fig. 1(b)). The darker color of reaction product was responsible for the increased light absorption. Moreover, the results showed that the highest absorbance was observed by the blue color component, instead of green as the intensity one. This was attributed that the red was the complementary color of green [25] and thus the highest absorbance of this color among others was found. These results showed a good agreement with a conventional spectrophotometric method, for which strong absorption at $880 \mathrm{~nm}$ has been described in the region of red wavelength [23].

In the phosphate reaction, the interaction between phosphate, ammonium molybdate and potassium antimonyl tartrate produced the phosphorus molybdenum heteropoly acid, which was then reduced by ascorbic acid to form molybdenum blue [23]. Therefore, the content of ammonium molybdate considerably impacted on the colorimetric reaction. Its effect of ammonium molybdate on the intensity was existed on Fig. 2.
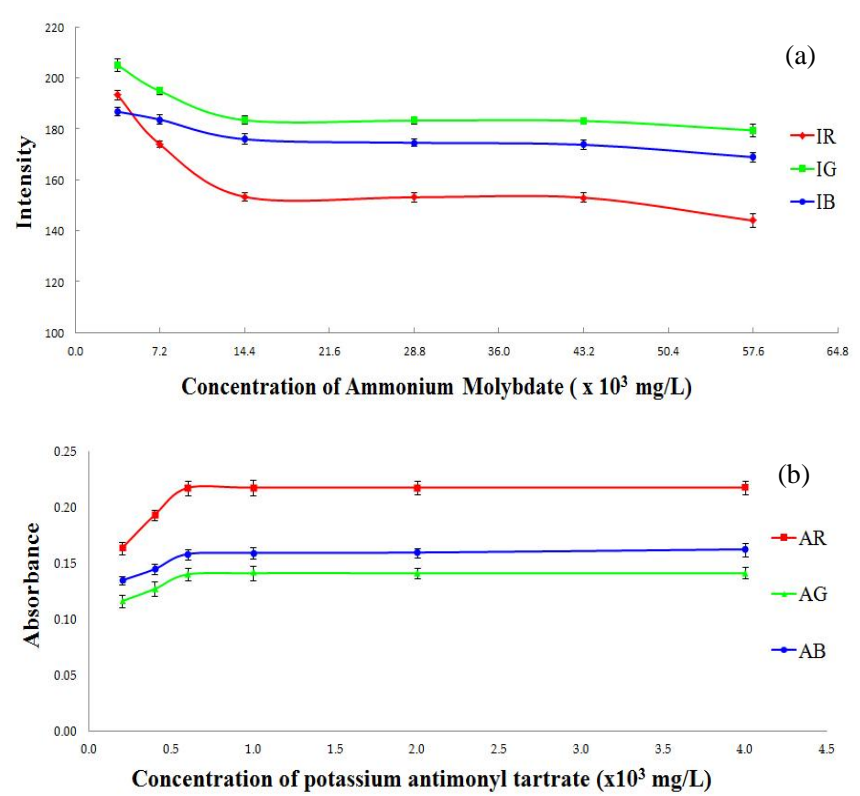

Fig. 2. The effect of ammonium molybdate concentration in sensor on (a) the RGB intensity and (b) absorbance for $1 \mathrm{mg} / \mathrm{L}$ phosphate detection.

The same situation as potassium antimonyl tartrate was displayed in Fig. 2 for ammonium molybdate effect. It was obvious that the color intensity was decreased with the increasing ammonium molybdate concentration in the range of $3.6-14.4 \mathrm{~g} / \mathrm{L}$. It was attributed to the rising up of the reaction intermediate of phosphorus molybdenum heteropoly acid leading to the increment of the molybdenum blue formation as the result. However, the increase in the ammonium molybdate more than $14.4 \mathrm{~g} / \mathrm{L}$ was not affected to the color intensity. It was owing to the phosphate ion used up.

The molecular absorption of color products was also considered in this section by calculating absorbance at each molybdate concentration using an equation previously described (1). The absorption of red light was found greater than blue and green light (Fig. 2(b)). It was also showed sharply alteration both in absorbance and intensity, indicating the high sensitivity property of this color.

As mentioned above, the other reagent involved in the reaction was ascorbic acid, which acted as the reducing agent in the mechanism. In this study, the effect of ascorbic acid concentration in the range of $0.88-10.56 \mathrm{~g} / \mathrm{L}$ was presented in Fig. 3.
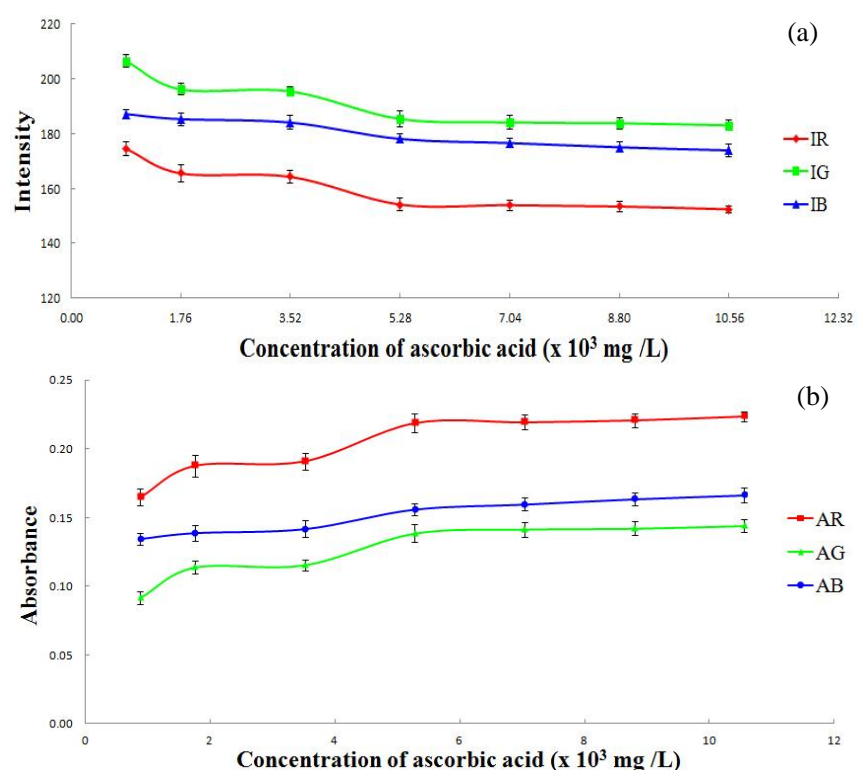

Fig. 3. The effect of ascorbic acid concentration in sensor on (a) the RGB intensity and (b) absorbance for $1 \mathrm{mg} / \mathrm{L}$ phosphate detection.
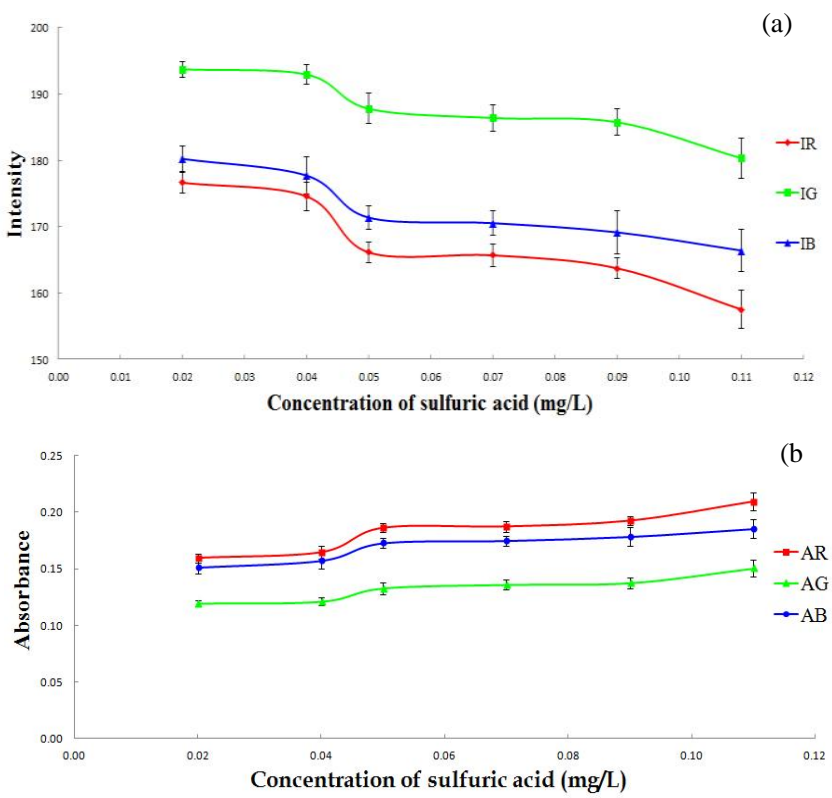

Fig. 4. The effect of sulfuric acid acid concentration in sensor on the RGB intensity for $1 \mathrm{mg} / \mathrm{L}$ phosphate detection.

The relationship between the ascorbic acid concentration and the product intensity/absorbance was similar to those obtained from other reagent, i.e. the RGB intensity was decreased with increasing concentration, while the absorbance was in opposite way (Fig 3(a) and 3(b)). The reason was possessed by the strengthen product color. However, its intensity and absorbance began to be steadfast after $5.28 \mathrm{~g} / \mathrm{L}$, so the optimum ascorbic acid was selected at 
that level with the sense of cost-effectiveness.

Since the reaction could be occurred in only acidic condition [22], the amount of acid addition was significant and was determined in this research for the scope of $0.02-$ $0.11 \mathrm{mg} / \mathrm{L}$. The results were shown in Fig. 4.

Obviously, the curve in Fig. 4(a) and 4(b) could be divided into 2 ranges, i.e. the high level of RGB color and the low one. The critical point was found at $0.05 \mathrm{mg} / \mathrm{L}$ sulfuric acid. This was explained by experimental results: when the acid concentration was lower than $0.05 \mathrm{mg} / \mathrm{L}$, the color of sensor was changed to blue-green, even no phosphate addition. This is way the concentration of green and blue was high at low concentration of acid applied. Since the detection greatly depended on the color change, it was not proper to use the acid at too low concentration. Thus, the optimum concentration of sulfuric acid was recommended at 0.05 $\mathrm{mg} / \mathrm{L}$.

\section{B. Effect of Reaction Time}

One of parameters affecting to the image color was the stability of the reaction product. It steadiness immensely depended on the reaction time. Thereby, the effect of time was investigated in this study in the range of 0.5-60 min to keep the color stabilize and rise up a system efficiency in terms of a speed analysis. The results were shown in Fig. 5(a) and 5(b).
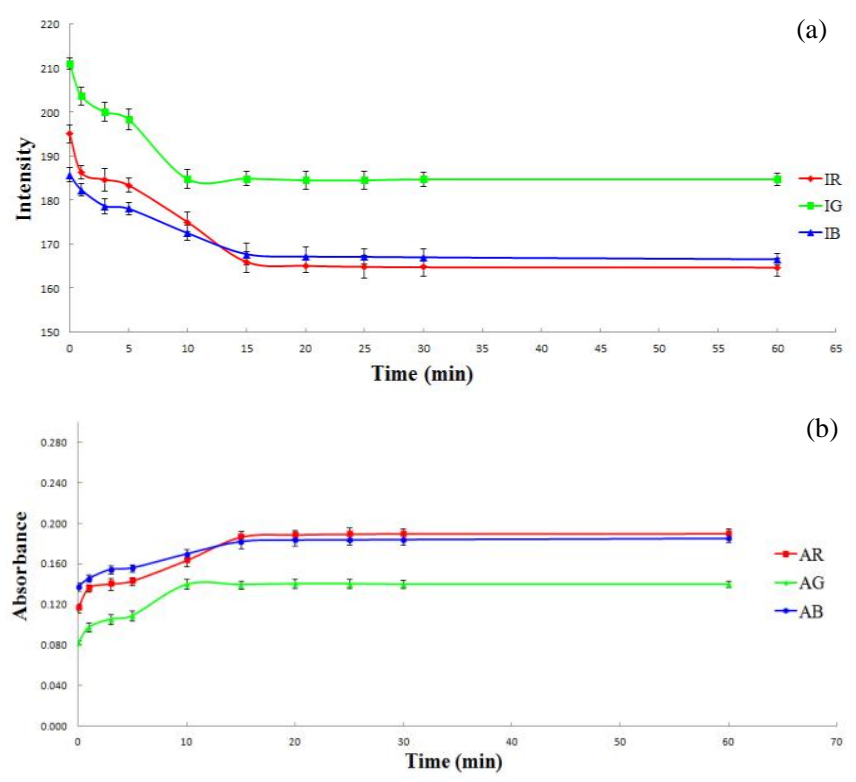

Fig. 5. The effect of reaction time on (a) the RGB intensity and (b) absorbance for $1 \mathrm{mg} / \mathrm{L}$ phosphate detection.

Like previous section, the intensity was inversely proportional to the reaction time in the range of $0-15 \mathrm{~min}$ and the line became constant after $15 \mathrm{~min}$, since the reagent or phosphate was used up. Consequently, the appropriate reaction time should be selected at $15 \mathrm{~min}$ in order to maximize the product concentration. The conclusion was supported by the water quality standard method, which suggested 10 to $15 \mathrm{~min}$ for the reaction undergoing [23].

\section{Preliminary Application with Water Sample}

Three surface waters were sampled from the water pond within university campus to preliminary test the efficiency of the developed sensor compared with the traditionally analytical technique [23]. Insignificant difference of $3.63 \%$ for a measured phosphate concentration was observed by spectrophotometer and the proposed sensor. This was indicated the possibility of sensor application for phosphate measurement and it deserved to study with more kind of water sample.

\section{CONCLUSION}

A PVA colorimetric polymer sensor was successfully developed for phosphate detection by entrapping color reagents within the PVA hydrogel matrix. The sensor was fabricated within a micro-PCR tube making it easy to transport and use. A small volume of the sample solution could be directly added into the tube during testing. The digital camera installed in the laptop computer could be used to detect the color of the reaction product rather than traditional human eye, providing more accurate and reliable results. With low amount of chemical used, it indicated the potential of the method in the aspect of cost-effectiveness.

\section{ACKNOWLEDGMENT}

The authors would like to express their thanks to Center of Excellence on Hazardous Substance Management (HSM), Chulalongkorn University for seeding money to initiate the project. The appreciation would be passed to the Faculty of Technology and Environment, Prince of Songkla University, Phuket Campus for funding, equipment and other resource supplies.

\section{REFERENCES}

[1] I. Zohar, A. Shaviv, M. Young, C. Kendall, S. Silva, and A. Paytan, "Phosphorus dynamics in soils irrigated with reclaimed waste water or fresh water - A study using oxygen isotopic composition of phosphate," Geoderma, vol. 159, pp. 109-121, 2010.

[2] W. L. Lindsay, Phosphates. Chemical Equilibria in Soils, USA: Wiley Interscience publication, 1979.

[3] A.Y. E. Sayed, Y. Z. Hussein, and M. A. Mohammed, "Simultaneous determination of phosphate and silicate in detergents and waters by first-derivative spectrophotometry," Analyst, vol. 126, pp.1810-1815, 2001.

[4] M. Batté, B. Koudjonoua, P. Laurent, L. Mathieub, J. Coalliera, and M Prévosta, "Biofilm responses to ageing and to a high phosphate load in a bench-scale drinking water system," Water Res., vol. 37, pp. 1351-1361, 2003.

[5] B. Shyla, Mahadevaiah, and G. Nagendrappa, "A simple spectrophotometric method for the determination of phosphate in soil, detergents, water, bone and food samples through the formation of phosphomolybdate complex followed by its reduction with thiourea,' Spectrochim. Acta, Part A, vol. 78, pp. 497-502, 2011.

[6] M. Snezana, Z. Valentina, O. Mirjana, T. Snezana, and P. Aleksandra, "Catalytic kinetic spectrophotometric method for determination of phosphate ion," Chin. J. Chem., vol. 25, pp. 531-534, 2007.

[7] A. E. Durrant, M. D. Scrimshaw et al., "Lester. Review of feasibility of recovering phosphate from wastewater for use as a raw material by the phosphate industry," Environ. Technol., vol. 20, pp. 749-758, 1999.

[8] R. P. Mihajlovic, V. M. Kaljevic, M. P. Vukasinovic, L. V. Mihajlovic, and I. Pantic, "Spectrophotometric method for the determination of phosphorus in natural waters using the bismuth-phosphomolybdate complex," Water SA, vol. 33, no. 4, pp. 513-517, 2007.

[9] N. Nakatani, D. Kozaki, W. Masuda, N. Nakagoshi, K. Hasebe, M. Mori, and K. Tanaka, "Simultaneous spectrophotometric determination of phosphate and silicate ions in river water by using ion-exclusion chromatographic separation and post-column derivatization," Anal. Chim. Acta, vol. 619, pp. 110-114, 2008.

[10] S. Li, F. Liu, F. Zheng, Y. Zuo and X. Huang, "Effects of nitrate addition and iron spectiation on trace element transfer in coastal food webs under phosphate and iron enrichment," Chemosphere, vol. 91, pp. 1486-1494, 2013. 
[11] A. Attour, M. Touati, M. Tlili, Ben, M. Amor, F. Lapicque, and J.-P. Leclerc, "Influence of operating parameters on phosphate removal from water by electrocoagulation using aluminum electrodes," Sep. Purif. Technol., vol. 123, pp. 124-129, 2014.

[12] J. L. Haberer and J. A. Brandes. "A high sensitivity, low volume HPLC method to determine soluble reactive phosphate in freshwater and saltwater," Mar. Chem., vol. 82, pp. 185-196, 2003.

[13] E.L.C. Silveira, L. B. de Caland, and M. Tubino, "Simultaneous quantitative analysis of the acetate, formate, chloride, phosphate and sulfate anions in biodiesel by ion chromatography," Fuel, vol. 124, pp. 97-101, 2014.

[14] Y. Y. Maruo, J. Nakamura, M. Uchiyama, M. Higuchi, and K. Izumi, "Development of formaldehyde sensing element using porous glass impregnated with Schiff's reagent," Sens. Actuators, B, vol. 129, pp.544-550, 2008.

[15] X. Wang, Y. Li, X. Li, J. Yu, S. S. Al-Deyab, and B. Ding, "Equipment-free chromatic determination of formaldehyde byutilizing pararosaniline-functionalized cellulose nanofibrous membrane," Sens. Actuators, B, vol. 203, pp. 333-339, 2014

[16] O. Bunkoed, F. Davis, P. Kanatharana, P. Thavarungkul, and S. P. J. Higson, "Sol-gel based sensor for selective formaldehyde determination," Anal. Chim. Acta, vol. 659, no. 1-2, pp. 251-257, 2010.

[17] L. Feng, Y. Liu, X. Zhou, and J. Hu, "The fabrication and characterization of a formaldehyde odor sensor using molecularly imprinted polymers," J. Colloid Interface Sci., vol. 284, no. 2, pp.378-382, 2005.

[18] E. G. Crispim, J. F. Piai, and A. R. Fajardo, "Hydrogels based on chemically modified poly(vinyl alcohol) (PVA-GMA) and PVA-GMA/chondroitin sulfate: Preparation and characterization," Express Polym. Lett., vol. 6, pp. 383-395, 2012.

[19] L. Wu and C. S. Brazel, "Modifying the release of proxyphylline from PVA hydrogels using surface crosslinking," Int. J. Pharm., vol. 349, pp 144-151, 2008.

[20] P. Kueseng, C. Thammakhet, P. Thavarungkul, and P. Kanatharana, "Multiwalled carbon nanotubes/ cryogel composite, a new sorbent for determination of trace polycyclic aromatic hydrocarbons," Microchem. J., vol. 96, no. 2, pp. 317-323, 2010.
[21] F. M. Plieva, M. Karlsson, M.-R. Aguilar, D. Gomez, S. Mikhalovsky, I. Y. Galaev, and B. Mattiasson, "Pore structure of macroporous monolithic cryogels prepared from poly(vinyl alcohol)," J. Appl. Polym. Sci., vol. 100, pp. 1057-1066, 2006.

[22] Y. Liu, X. Cao, R. Hua, Y. Wang, Y. Liu, C. Pang, and Y. Wang, "Selective adsorption of uranyl ion on ion-imprinted chitosan/PVA cross-linked hydrogel," Hydrometallurgy, vol. 104, no. 2, pp. 150-155, 2010.

[23] APHA, AWWA and WEF, Standard Methods for the Examination of Water and Wastewater, 21st edition, Washington DC, USA, 2005.

[24] A. Choodum and N. NicDaied, "Digital image-based colourimetric tests for amphetamine and methylamphetamine," Drug Test. Anal., vol. 3, no. 5, pp. 277-28, 2011.

[25] J. Roker and D. Chak Exploring Complementary Colours, Melbourne, Australia: Zart Art, 2008.

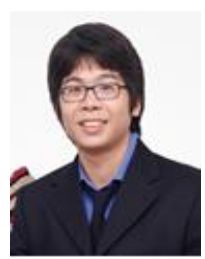

Worawit Wongniramaikul was born in 1979. He received a doctor of philosophy in environmental management from Chulalongkorn University, Thailand in 2007. He has been working at Prince of Songkla University, Phuket Campus, Thailand since 2007.

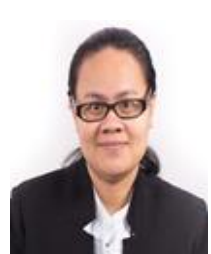

Aree Choodum was born in 1979. She received a doctor of philosophy in chemistry from Prince of Songkla University, Thailand in 2012. She has been working at Prince of Songkla University, Hat Yai Campus, Thailand since 2012. In 2014 she moved to Phuket Campus. She currently an Associate Professor at Faculty of Technology and Environment. 